\title{
Identification of C38 colon adenocarcinoma growth under bevacizumab therapy and without therapy
}

\author{
Johanna Sápi*, Dániel András Drexler ${ }^{\dagger}$,Zoltán Sápi ${ }^{\ddagger}$ and Levente Kovács* \\ * Óbuda University, Institute of Applied Informatics, Physiological Controls Group, \\ Bécsi út 96/B H-1034 Budapest, Hungary \\ Email: sapi.johanna@phd.uni-obuda.hu, kovacs.levente@nik.uni-obuda.hu \\ ${ }^{\dagger}$ Budapest University of Technology and Economics, Department of Control Engineering and Information Technology \\ Magyar tudósok krt 2. H-1117 Budapest, Hungary \\ Email: drexler@iit.bme.hu \\ $\ddagger$ Semmelweis University, 1st Department of Pathology and Experimental Cancer Research, \\ Üllői út 26. H-1085 Budapest, Hungary \\ Email: sapi.zoltan.dr@gmail.com
}

\begin{abstract}
Model identification allows to design different control strategies for antiangiogenic cancer therapy, and create model-based treatment protocols. These model-based protocols can be more effective than the current ones, since they provide individual treatment for the patients. The aim of this paper is to investigate $\mathrm{C38}$ colon adenocarcinoma growth in three different cases: (1) tumor growth without therapy, (2) tumor growth with one Avastin dose for a 18-day therapy $(10 \mathrm{mg} / \mathrm{kg})$, (3) tumor growth with one-tenth dose of control Avastin dose spread over 18 days. Parametric model identification was carried out for these three cases and the relationship between the measured tumor attributes (volume, mass and vascularization) was analyzed. Effect of low-dose therapy was also examined.
\end{abstract}

\section{INTRODUCTION}

The key of scientific success in every field nowadays depends on interdisciplinary design [1]. Medical treatment is not an exception either, engineers and doctors have to work together to find more effective solutions in healing. Beside classical cancer treatments (surgical oncology, radiotherapy, chemotherapy) a new approach have appeared called targeted molecular therapies (TMTs). The main goal of these treatments is to find a cancer specific mechanism which can be selectively inhibited, thus the therapy do not have impact to healthy cells (virtually do not have significant side effects). However finding an appropriate medication is not enough, the effectiveness of the treatment strongly depends on the drug administration as well. Opposed to general protocols, precise, individual treatment have to be developed - that can be executed by closed loop controllers.

Antiangiogenic therapy is a type of TMTs, which inhibits angiogenesis. Angiogenesis is the process of forming new blood vessels; normally it occurs in the human body only at specific times (e.g. in case of wound healing). Tumor cells can break through this strict control and become able to form own blood vessels, which is essential for survival after a certain tumor size ( $1-2 \mathrm{~mm}$ diameter). In antiangiogenic therapy, the patients get angiogenic inhibitor, which inhibits tumor-induced angiogenesis, hence tumors will not be able to form new blood vessels by sprouting from existing vessels. There are several angiogenic inhibitors used in clinical practice, which act in different molecular ways [2]. Widely used inhibitors in cancer therapies are endostatin [3] and bevacizumab [4]. The paper is focusing on the identification of tumor growth using the latter bevacizumab (Avastin) therapy.

The paper is organized as follows. In Section II, we give a brief overview of the phases of the experiment, and the methods used in the experiment are described. Analysis of the experimental results are shown is Section III, where three topics are discussed: (1) C38 colon adenocarcinoma growth identification with and without Avastin therapy, (2) Finding the relationship between tumor volume, mass and vascularization, (3) Finding the effective dosage for optimal therapy. The paper ends in Section IV with the conclusion.

\section{MATERIALS AND METHODS}

This experiment is a collaboration between Physiological Controls Group, Óbuda University (Budapest, Hungary) and 1st Department of Pathology and Experimental Cancer Research, Semmelweis University (Budapest, Hungary). The identification procedure was supplied by several measurements planned in a rigorous way. The aim of the experiment is to create and validate a clinically relevant tumor growth model, focusing on the effect of angiogenesis. Model identification allows one to design different control strategies for antiangiogenic cancer therapy, and create model-based treatment protocols. These model-based protocols can be more effective than the current ones, since they provide individual treatment for the patients.

\section{A. Overview of the phases}

In Phase I, we have investigated tumor growth without therapy with two types of mouse tumor. 12 mice were transplanted subcutaneously with C38 colon adenocarcinoma, and 11 mice were injected intramuscularly with B16 melanoma. Results of this phase can be found in [5]. Phase II was the toxicology investigation of the applied angiogenic inhibitor (Avastin); there was no tumor implantation into mice in this 
C38 colon adenocarcinoma

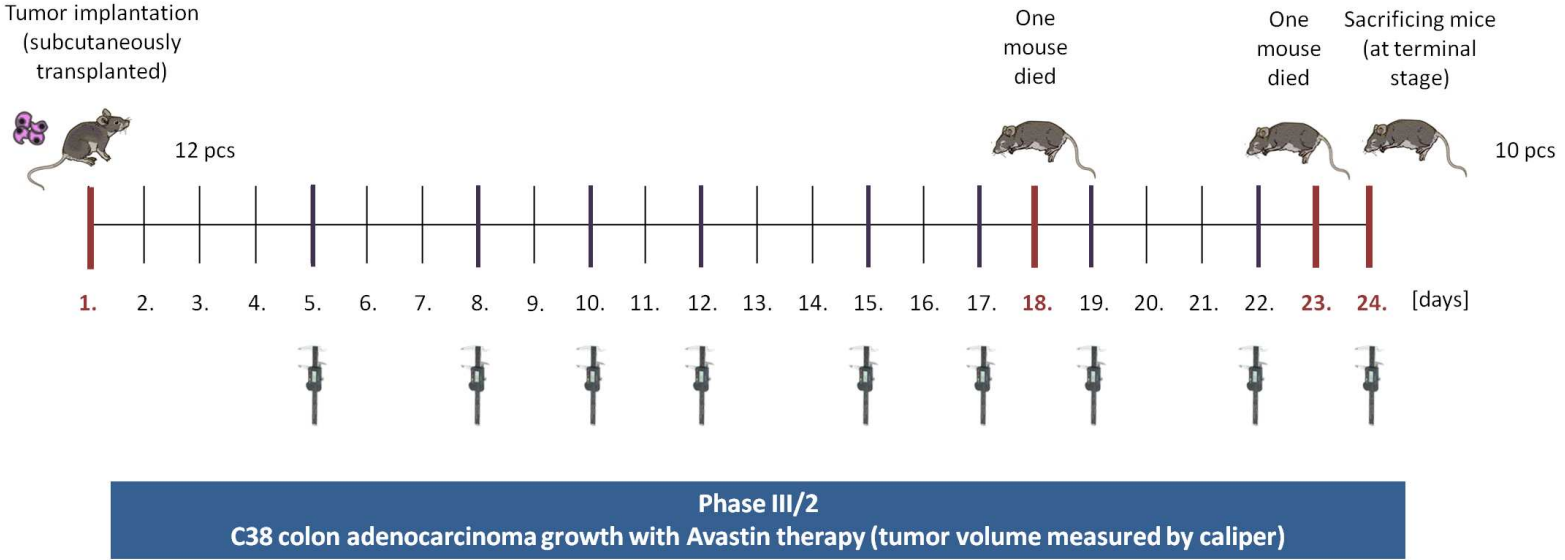

Controlgroup

Avastin administration
Tumor implantation $\quad 200 \mu \mathrm{g}$ Avastin \& $455 \mu \mathrm{l} 0.9 \% \mathrm{NaC}$

(subcutaneously $\quad 200 \mu \mathrm{g}$ Avastin \& $455 \mu \mathrm{l} 0.9 \% \mathrm{NaCl}$ solution

transplanted) (10 mg per kg body weight $\quad$ Sacrificing mice

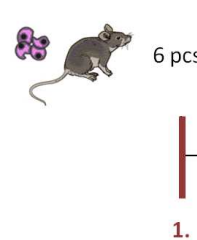
intraperitoneally)
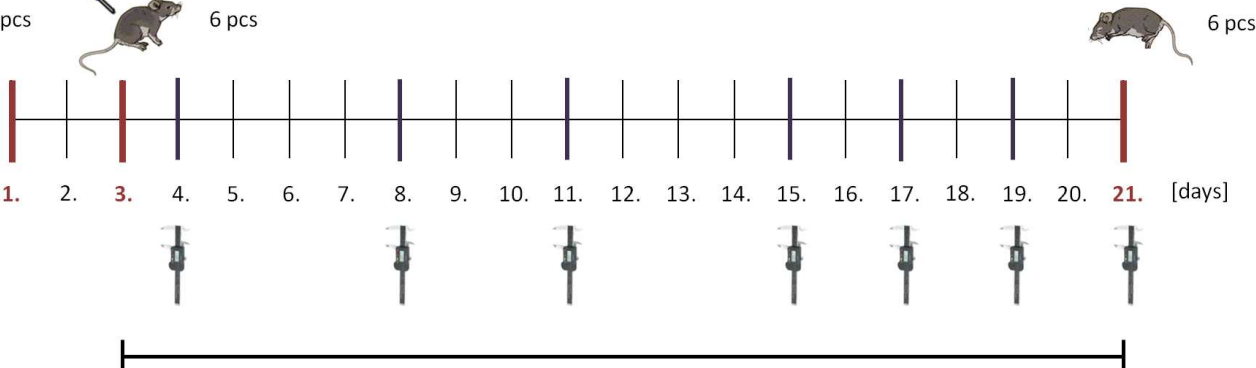

18 days Avastin treatment

(time was specified according to Phase III/1)

Case group

Avastin administration every day
$\begin{gathered}\text { Tumor implantation } \\ \text { (subcutaneously } \\ \text { transplanted) }\end{gathered}$
(one-tenth dose of control dose
intraperitoneally spread over 18 days)

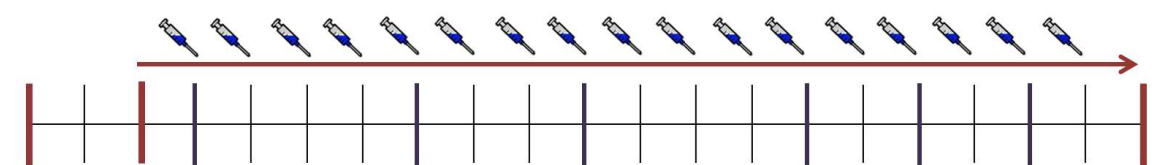

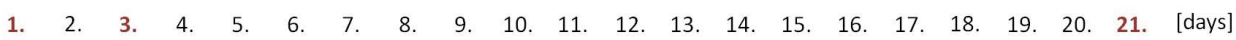

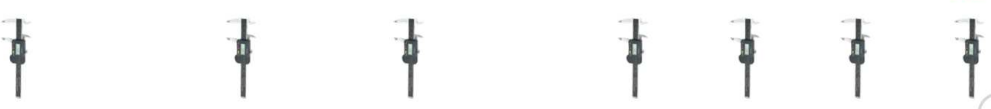

Notation
if Tumor volume

measured by caliper

Fig. 1. Detailed description and summary of the phases of the experiment. 
phase. We monitored the vital parameters of 4 mice, and we could exclude any serious toxic effects or lethality regarding the usage of Avastin. In Phase III, we have investigated C38 colon adenocarcinoma growth with Avastin therapy. In the first subphase (Phase III/1), we have examined the optimal administration of the angiogenic inhibitor, particularly with regard to the quantity of the solvent (with 10 mice). In the second subphase (Phase III/2), we created two groups. Control group (6 mice) received Avastin in one dose according to the protocol, case group (12 mice) received less dose every day (see Section II.C).

Relevant phases according to this study can be found in Fig. 1

\section{B. Tumor implantation}

A piece of C38 colon adenocarcinoma was transplanted subcutaneously in the recipient animal.

\section{Antiangiogenic therapy (Avastin administration)}

As we have mentioned above, endostatin and bevacizumab are typical angiogenic inhibitors in cancer treatment. Endostatin is an endogenous collagen, which inhibits cell migration, cell proliferation and survival of endothelial cells [3]. In contrast, bevacizumab (Avastin) [6] is an exogenous inhibitor, which inhibits the biologic activity of human VEGF [7].

Recommended administration of Avastin is one $5-10 \mathrm{mg} / \mathrm{kg}$ dose for $2-3$ weeks [6]. We have administered $10 \mathrm{mg}$ per $\mathrm{kg}$ body weight intraperitoneally, which means $200 \mu \mathrm{g}$ Avastin per a mouse since the mass of the mice in the experiment was $20 \mathrm{~g}$. This dose was used for a 18-day treatment.

In Phase I there was no Avastin administration. In Phase III/2 the control group received $200 \mu \mathrm{g}$ Avastin (with $455 \mu \mathrm{l}$ $0.9 \% \mathrm{NaCl}$ solution) in one dose intraperitoneally in the $3 \mathrm{rd}$ day. In Phase III/2 the case group received one-tenth dose of control dose intraperitoneally speared over 18 days. It means that a case mouse received $1.11 \mu \mathrm{g}$ Avastin (with $45 \mu \mathrm{l} 0.9 \%$ $\mathrm{NaCl}$ solution) every day for 18 days. Avastin administration for the case group started at the $3 r d$ day as well.

\section{Tumor volume measurement}

Tumor volume measurement cannot happen right after tumor implantation. First, the subcutaneously transplanted piece of tumor has to disintegrate, and after that the new tumor colony (what we want to measure) can begin to grow from the disintegrated tumor cells. This process takes 4-5 days. The first measurement in Phase I occurred on the 5th day, in Phase III/2 it occured on the $4 t h$ day. Tumor diameters (width, length) could be measured with a digital caliper during the experiment because of the subcutaneous localization of the tumor. The third dimension of the tumor (height) was approximated with the length multiplied by $2 / 3$. Assuming ellipsoid shape, the tumor volume in $\mathrm{mm}^{3}$ was calculated by the formula:

$$
V=\frac{4}{3} \cdot \pi \cdot \frac{l}{2} \cdot \frac{w}{2} \cdot \frac{l}{3},
$$

where $l$ is tumor length, $w$ is tumor width, and $h$ is tumor height.

\section{E. Sacrificing mice}

In Phase III/2 mice were sacrificed after the 18-day treatment, on the $21 s t$ day of the experiment. After sacrificing the mice, the tumors were removed, and their volume and mass were measured. After that, tumors were cut into two pieces: one piece was stored in formalin, and the other piece was freezed using liquid nitrogen.

\section{F. Tumor sample processing}

Tumor morphology was investigated using standard Haematoxylin Eosin (H\&E) staining [8] with the samples stored in formalin. The freezed samples were used to create frozen sections [9] of the tumor. Tumor vasculation was stained in vitro using CD31 antibody immunohistochemistry staining [10] on frozen sections. After staining, fluorescence pictures were done with confocal microscope and from these pictures vascularization area was calculated by ImageJ [11] software.

\section{ANALYSIS OF THE EXPERIMENTAL RESULTS}

\section{A. Experimental data}

In Phase I 12 mice were implanted with C38 colon adenocarcinoma. One of them died on the 18th day, and another one on the 23th day. Thus 10 mice were sacrificed on the $24 t h$ day of the experiment. In Phase III/2 the control group contained 6 mice, the case group contained 12 mice; all mice were implanted with $\mathrm{C} 38$ colon adenocarcinoma. No mice died during the experiment, therefore 6 contol and 12 case mice were sacrificed after the 18-day Avastin treatment, on the 21th day of the experiment.

\section{B. C38 colon adenocarcinoma growth identification with and without Avastin therapy}

In tumor growth there are two main processes which take place. The first process, actually the engine of tumor growth is the vascular growth; new blood vessels are indispensable for the tumor to pick up enough nutrients and oxygen. With the support of vasculature, tumor mass growth can occur as the second process. Taking into account these two dynamics behind tumor growth, we are seeking for a second order system for identification. The second order system has two exponential functions in its response, thus parametric identification was carried out by fitting a curve with two exponential functions. The curve was fitted to the average tumor volume of each mice at the measurement points (days). Since the system of tumor growth is unstable, identification of the system is difficult.

Result of the parametric identification in the case of C38 colon adenocarcinoma growth without antiangiogenic therapy (Phase I) was:

$$
y_{p I}(t)=29020 \cdot \exp (0.29788 t)-29010 \cdot \exp (0.29789 t)
$$

Time constants of the system are $T_{1}=3.3570$ days, $T_{2}=$ 3.3568 days. 

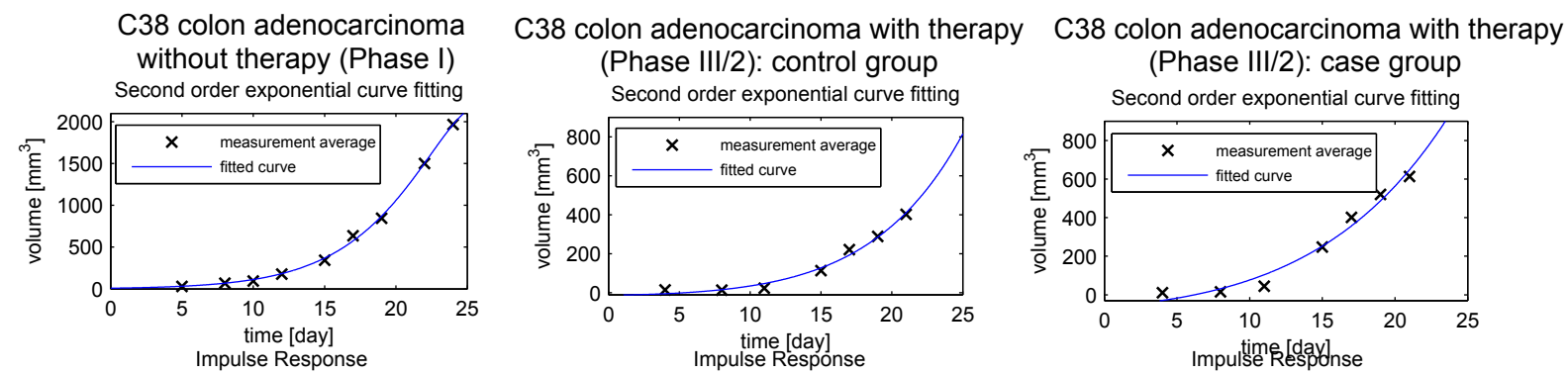
without therapy (Phase I)
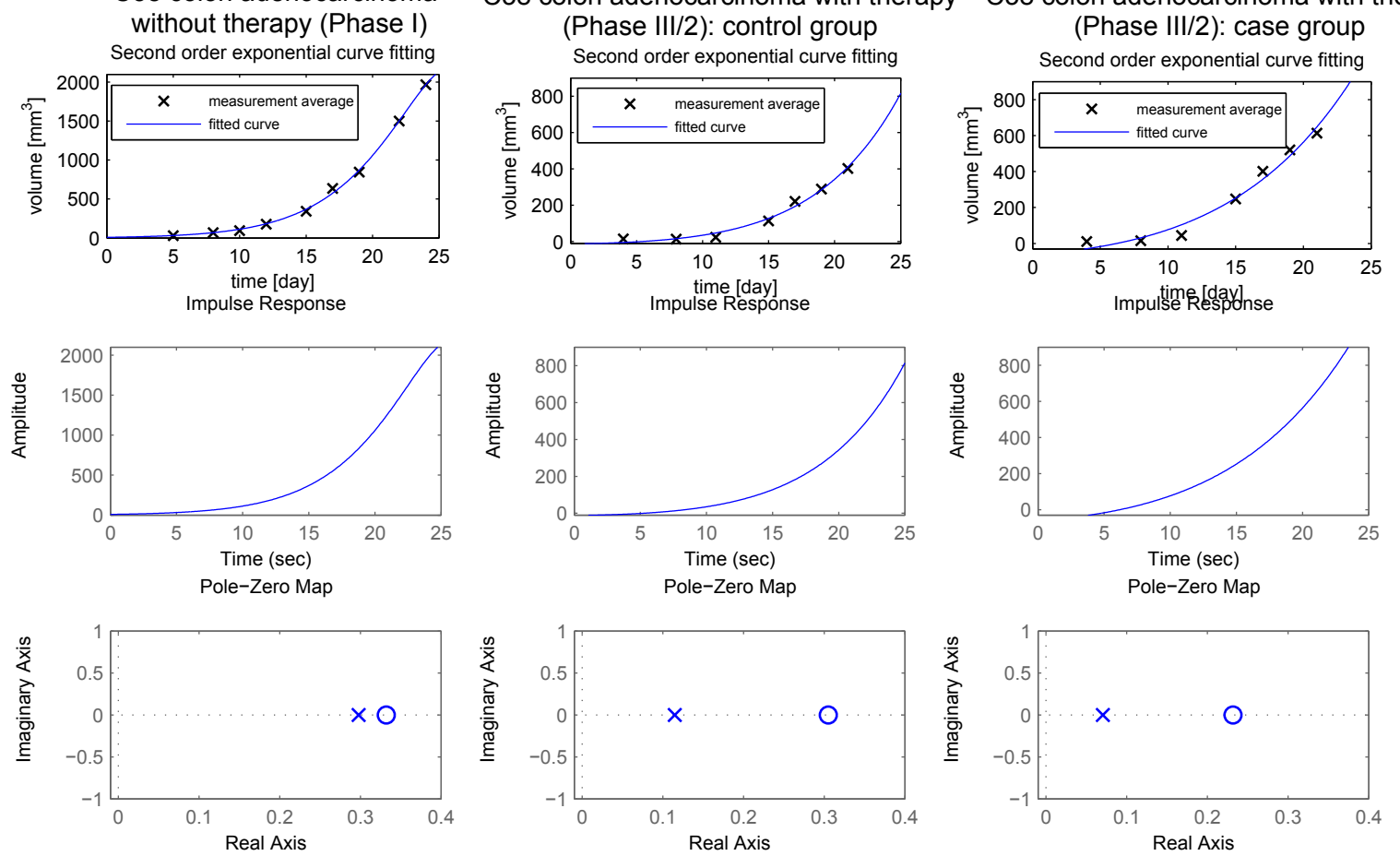

Fig. 2. Comparison of C38 colon adenocarcinoma growth in three different cases. In Phase I, tumor growth was investigated without antiangiogenic therapy; in Phase III/2, control group members received one $200 \mu \mathrm{g}$ Avastin dose for a 18-day therapy; in Phase III/2, case group members received $1.11 \mu \mathrm{g}$ Avastin every day for 18 days. The first row shows the second order exponential curve fitting for the average of measurement points; in the second row one can see the impulse response of the identificated systems; while the third row shows the poles and zeros of the identificated systems.

Result of the parametric identification in the case of $\mathrm{C} 38$ colon adenocarcinoma growth with Avastin therapy (Phase III/2), control group was:

$$
\begin{array}{r}
y_{\text {pIII } / 2 \text { control }}(t)= \\
2.28171 \cdot 10^{6} \cdot \exp (0.114578 t) \\
+2.28170 \cdot 10^{6} \cdot \exp (0.114579 t)
\end{array}
$$

Time constants of the system are $T_{1}=8.7277$ days, $T_{2}=$ 8.7276 days.

Result of the parametric identification in the case of C38 colon adenocarcinoma growth with Avastin therapy (Phase III/2), case group was:

$$
\begin{array}{r}
y_{p I I I / 2 \text { case }}(t)= \\
1.37190 \cdot 10^{6} \cdot \exp (0.07045 t) \\
-1.37196 \cdot 10^{6} \cdot \exp (0.07044 t)
\end{array}
$$

Time constants of the system are $T_{1}=14.1935$ days, $T_{2}=$ 14.1950 days.

Comparison of the results in the three different cases can be found in Fig. 2. The coefficients of the exponential functions are positive in every cases, reflecting the unstability of the system (as it is required from a tumor growth model). As one can see, in every cases parametric identification resulted in almost identical time constants; however, the usage of two exponential function is not pointless. From the physiological point of view there are two concrete dynamics which have to be modeled. The engineering interpretation of this result is an integrator series, which means that the change of the first state variable (which is also the output of the system, that is the tumor volume) depends on the second state variable (vascularization). In addition, the change of the second state variable depends on the input. Both interpretations are physiologically correct.

From the fitted curves, transfer function of the models can be calculated.

Transfer function of C38 colon adenocarcinoma growth without antiangiogenic therapy (Phase I) resulted in:

$$
W_{p I}(t)=\frac{8.715 s-2.895}{s^{2}-0.5958 s+0.08874}
$$

Poles of the system are $p_{p I, 1}=0.29788, p_{p I, 1}=0.29789$.

Transfer function of C38 colon adenocarcinoma growth with Avastin therapy (Phase III/2), control group resulted in:

$$
W_{\text {pIII/2control }}(t)=\frac{-12.34 s+3.764}{s^{2}-0.2292 s+0.01313}
$$

Poles of the system are $p_{p I I I / 2 \text { control }, 1}=0.11457$, $p_{\text {pIII } / 2 \text { control }, 2}=0.11458$.

Transfer function of C38 colon adenocarcinoma growth with Avastin therapy (Phase III/2), case group resulted in:

$$
W_{p I I I / 2 \text { case }}(t)=\frac{-61.79 s+14.33}{s^{2}-0.1409 s+0.004963}
$$




\begin{tabular}{|c|c|c|c|}
\hline & $\begin{array}{l}\text { C38 colon adenocarcinoma } \\
\text { without therapy (Phase I) }\end{array}$ & $\begin{array}{c}\text { C38 colon adenocarcinoma } \\
\text { with therapy (Phase III/2): } \\
\text { control group }\end{array}$ & $\begin{array}{c}\text { C38 colon adenocarcinoma } \\
\text { with therapy (Phase III/2): } \\
\text { case group }\end{array}$ \\
\hline \multirow{4}{*}{$\begin{array}{l}\text { tumor volume } \\
\text { and mass }\end{array}$} & $R=0.941$ & $R=0.955$ & $R=0.913$ \\
\hline & $R^{2}=0.885$ & $R^{2}=0.911$ & $R^{2}=0.834$ \\
\hline & $p<0.0001$ & $p=0.003$ & $p<0.0001$ \\
\hline & positive correlation & positive correlation & positive correlation \\
\hline \multirow{4}{*}{$\begin{array}{l}\text { tumor volume } \\
\text { and vascularization }\end{array}$} & $R=0.269$ & $R=-0.619$ & $R=0.078$ \\
\hline & $R^{2}=0.072$ & $R^{2}=0.383$ & $R^{2}=0.006$ \\
\hline & $p=0.453$ & $p=0.190$ & $p=0.811$ \\
\hline & positive correlation & negative correlation & positive correlation \\
\hline \multirow{4}{*}{$\begin{array}{c}\text { tumor mass } \\
\text { and vascularization }\end{array}$} & $R=0.198$ & $R=-0.780$ & $R=0.150$ \\
\hline & $R^{2}=0.039$ & $R^{2}=0.608$ & $R^{2}=0.023$ \\
\hline & $p=0.584$ & $p=0.067$ & $p=0.641$ \\
\hline & positive correlation & negative correlation & positive correlation \\
\hline
\end{tabular}

Fig. 3. Linear regression analysis for tumor volume - tumor mass, tumor volume - vascularization, and tumor mass - vascularization pairs. In Phase I, tumor growth was investigated without antiangiogenic therapy; in Phase III/2, control group members received one $200 \mu \mathrm{g}$ Avastin dose for a 18 -day therapy; in Phase III/2, case group members received $1.11 \mu \mathrm{g}$ Avastin every day for 18 days. $R$ is the Pearson correlation coefficient, $R^{2}$ is the coefficient of determination, $p$ is the ANOVA significance value (level of significance is $p=0.05$ ).

Poles of the system are $p_{p I I I / 2 \text { case }, 1}=0.07044$, $p_{\text {pIII } / 2 \text { case }, 2}=0.07045$.

From the poles of the systems (third row of Fig. 2) we can conclude that each system is unstable. To verify the goodness of the created transfer functions, we plotted the impulse response of each transfer function (second row of Fig. 2 ), which shows quite similar result to the curve fitting (first row of Fig. 2).

\section{Finding the relationship between tumor volume, mass and vascularization}

Three attributes of the lethal sized tumor were measured: tumor volume, tumor mass and vascularization. Relationship between these tumor attributes was investigated with linear regression analysis [12]. To decide wether the relationship is significant or not between two variables, we used the following statistics. Pearson correlation coefficient $(R)$ describes strength of the correlation (linear dependence) between the variables. Coefficient of determination $\left(R^{2}\right)$ tells how many percent of the variability in a data can be explained by the given statistical model (which is a linear model in every investigated cases). Using Analysis of Variance (ANOVA) test [13] we can decide that the regression analysis is valid or not (level of significance was chosen to $p=0.05$ ).

Results are summarized in Fig. 3. As one can see, the relationship between tumor volume and mass is significant and positive in all cases, which means that the larger the volume, the higher the mass. The third attribute, tumor vascularization shows interesting results.

On the one hand, vascularization does not have significant relationship with volume or mass in neither cases. The physiological explanation for this result is that in C38 colon adenocarcinoma there are several necrotic regions, thus the whole mass contains relatively few viable cells and vessels [14].

On the other hand in the case of Phase III/2 control group, tumor volume and tumor mass both have negative correlation with vascularization, however these relationships are not significant (tumor mass - vascularization have near-significant relationship). The possible explanation is the following [15]. In the case when angiogenesis occurs according to normal trigger, pro- and antiangiogenic factors have balance, consequently the newly formed vessels are normal with effective blood supply. However, in the case of tumor-induced angiogenesis, there is an extra proangiogenic factor produce due to hurried vessel forming, which result in abnormal vessels (high vascular permeability, poor perfusion) with inefficient blood supply. High interstitial fluid pressure can compress the vessels; thereafter abnormal tumor growth may continue, however delivery of therapeutic agents to the tumor is obstructed. Therefore, first abnormal vessels have to be normalized with the balance of pro- and antiangiogenic factors, thus vascular network can be restored. This creates the possibility of efficient therapeutic agent use.

In the case of Phase III/2 control group, mice received a big dose of Avastin according to the protocol. This resulted in a sudden preponderance of anti-factors; however, due to abnormal vessel network, the utilization of the antiangiogenic molecules was not effective. Despite the high dose, only a small fraction could be used. That is why larger tumors had fewer viable vessels in control group. In contrast in the case of Phase III/2 case group, mice received a small dose of Avastin, which - with the continuous, slow increase of antiangiogenic factors - enabled the normalization of blood vessels [16]; hence Avastin could be used more efficiently. 


\section{Finding the effective dosage for optimal therapy}

To compare the results of the three investigated cases (Phase I, Phase III/2 control group, Phase III/2 case group) we used statistical analysis. Tumor volume values, which were measured on the 21 st day in each phase, were compared. Before the usage of any statistical tests, one have to examine the normality and homogeneity of variance (homoscedasticity) of the distributions. Normality was investigated with onesample Kolmogorov-Smirnov test [17]; it was found that each sample has normal distribution $\left(p_{p I}=0.966, p_{p I I I / 2 \text { control }}=\right.$ $\left.0.999, p_{p I I I / 2 \text { case }}=0.608\right)$. Homogenity of variance was examined with Levene's test [18]; the sample variances are equal $(p=0.266)$.

After confirming normality and homoscedasticity, parametric statistical analysis can be used. With Analysis of Variance (ANOVA) test [13] more than two samples are comparable. ANOVA test was resulted in $p=0.038$ value, which means that we have to reject the null hypothesis according to which there are no differences between the means of the samples (using a $p=0.05$ level of significance). To find those samples, which have significantly differnet means, Tukey's honest significant difference (HSD) test was used as post hoc test. The results are the following. Phase I and Phase III/2 control group are significantly different $(p=0.034)$, which means that Avastin - administered according to the protocol - is an effective drug to reduce tumor volume. Phase III/2 control group and Phase III/2 case group are not significantly different ( $p=0.416)$, however Phase I and Phase III/2 case group are not significantly different $(p=0.227)$ either. This means that the effectiveness of the "continuous" (daily) $1 / 180$ dosage $(1.11 \mu \mathrm{g}$ relative to $200 \mu \mathrm{g})$ is comparable with the effectiveness of one large dose.

\section{CONCLUSION}

In our experiment three different cases were investigated. In Phase I, C38 colon adenocarcinoma growth was examined without antiangiogenic therapy; in Phase III/2, control group members received one $200 \mu \mathrm{g}$ Avastin dose for a 18-day therapy; in Phase III/2, case group members received 1.11 $\mu \mathrm{g}$ Avastin every day for 18 days (one-tenth dose of control dose intraperitoneally spread over 18 days). Parametric model identification was carried out for these three cases. Relationship between the measured tumor attributes (volume, mass and vascularization) was also analyzed.

We have found that the effectiveness of the "continuous" (daily) ${ }^{1 / 180}$ dosage $(1.11 \mu \mathrm{g}$ relative to $200 \mu \mathrm{g})$ is comparable with the effectiveness of one large dose. In addition, this is a short-term result (18-day treatment); predicted long-term results are more better, since the identified model for case group has slower dynamics (time constants of the system are approx. 14 days) than the identified model for control group (time constants of the system are approx. 8 days). Taking into account the physiological aspects as well, on the one hand, daily small dosage is better than one large dose, because it enables the normalization of blood vessels [16]; hence Avastin could be used more efficiently; on the other hand, if antiangiogenesis is persistent, it can completely destroy the vascular network, which leads to tumor necrosis (death of tumor) [15]. Furthermore, it does not have to be ignored that a considerably lower dose have considerably lower side-effects (or virtually nothing).

\section{ACKNOWLEDGMENT}

The authors gratefully acknowledge the financial support by the Óbuda University research fund. Levente Kovács was supported by the János Bolyai Research Scholarship of the Hungarian Academy of Sciences.

\section{REFERENCES}

[1] S. S. Vattam and A. K. Goel, "Semantically annotating research articles for interdisciplinary design," in Proceedings of the Sixth International Conference on Knowledge Capture, 2011, pp. 165-166. [Online]. Available: http://doi.acm.org/10.1145/1999676.1999707

[2] K. Dredge, A. G. Dalgleish, and J. B. Marriott, "Angiogenesis inhibitors in cancer therapy," Curr Opin Investig Drugs., vol. 4(6), pp. 667-674, 2003.

[3] M. S. O’Reilly, T. Boehm, Y. Shing, N. Fukai, G. Vasios, W. S. Lane, E. Flynn, J. R. Birkhead, B. R. Olsen, and J. Folkman, "Endostatin: An endogenous inhibitor of angiogenesis and tumor growth," Cell, vol. 88, pp. 277-285, 1997.

[4] L. M. Ellis and D. G. Haller, "Bevacizumab beyond progression: Does this make sense?" J Clin Oncol., vol. 26(33), pp. 5313-5315, 2008.

[5] J. Sápi, D. A. Drexler, I. Harmati, A. Szeles, B. Kiss, Z. Sápi, and L. Kovács, "Tumor growth model identification and analysis in case of C38 colon adenocarcinoma and B16 melanoma," SACI 2013 - 8th IEEE International Symposium on Applied Computational Intelligence and Informatics, pp. 303-308, 2013, Timisoara, Romania.

[6] Genentech, "Prescribing information of avastin (bevacizumab)," http://www.gene.com/download/pdf/avastin_prescribing.pdf, 2013, 01.08.2014.

[7] E. M. Agency, "Scientific discussion of avastin," http: //www.ema.europa.eu/docs/en_GB/document_library/EPAR_Scientific_Discussion/human/000582/WC500029262.pdf,

2005 , 01.08.2014.

[8] A. H. Fischer, K. A. Jacobson, J. Rose, and R. Zeller, "Hematoxylin and eosin staining of tissue and cell sections," CSH Protoc., vol. pdb.prot4986, 2008.

[9] C. S. Technology, "Immunohistochemistry frozen section protocol," http: //www.cellsignal.com/support/protocols/4060_ihcf.html, 01.02.2013.

[10] I. WORLD, "CD31 antibody staining protocol for immunohistochemistry," http://www.ihcworld.com/_protocols/antibody_protocols/ cd31_pharmingen.htm, 01.02.2013.

[11] ImageJ, "Image processing and analysis in Java," http://rsbweb.nih.gov/ ij/index.html, 01.02.2013.

[12] D. C. Montgomery, E. A. Peck, and G. G. Vining, Introduction to Linear Regression Analysis, fifth edition. John Wiley \& Sons, Inc., Hoboken, New Jersey, 2012.

[13] M. G. Larson, "Statistical primer for cardiovascular research. analysis of variance," Circulation, vol. 117, pp. 115-121, 2008.

[14] Y. J. Kamm, A. Heerschap, G. Rosenbusch, I. M. Rietjens, J. Vervoort, and D. J. Wagener, "5-fluorouracil metabolite patterns in viable and necrotic tumor areas of murine colon carcinoma determined by $19 \mathrm{f} \mathrm{nmr}$ spectroscopy," Magn Reson Med., vol. 53(13), pp. 2987-2993, 1996.

[15] A. Reinacher-Schick, M. Pohl, and W. Schmiegel, "Drug insight: antiangiogenic therapies for gastrointestinal cancers-focus on monoclonal antibodies," Nat Clin Pract Gastroenterol Hepatol., vol. 5(5), pp. 250 267, 2008.

[16] C. e. a. Willett, "Direct evidence that the vegf-specific antibody bevacizumab has antivascular effects in human rectal cancer," Nat Med., vol 10(2), pp. 145-147., 2004.

[17] H. Lilliefors, "On the Kolmogorov-Smirnov test for normality with mean and variance unknown,” J. Amer. Statist. Assoc., vol. 62(318), pp. 399402., 1967.

[18] J. Gastwirth, Y. Gel, and W. Miao, "The impact of Levene's test of equality of variances on statistical theory and practice," Statist. Sci., vol. 24(3), pp. 343-360., 2009 